\title{
Potential Adaptive Function for Altered Long-Term Potentiation Mechanisms in Aging Hippocampus
}

\author{
Katica Boric, ${ }^{1}$ Pablo Muñoz, ${ }^{1}$ Michela Gallagher, ${ }^{2}$ and Alfredo Kirkwood ${ }^{1}$ \\ ${ }^{1}$ Mind/Brain Institute and Department of Neurosciences and ${ }^{2}$ Department of Psychological and Brain Sciences, The Johns Hopkins University, Baltimore, \\ Maryland 21218
}

\begin{abstract}
Age-dependent alterations in the induction of long-term potentiation (LTP) are well documented, providing a likely neural basis for memory decline associated with aging. Studies of neural plasticity are also important to understand the neural basis of individual differences in aging, ranging from significant cognitive impairment to preservation of function on a par with younger adults. To examine the cellular mechanisms that distinguish such outcomes, we studied the induction of LTP in male outbred young and aged rats behaviorally characterized in hippocampal-dependent spatial learning. We evaluated, in vitro, the magnitude of NMDA receptor (NMDAR)dependent and -independent forms of LTP induced in the Schaffer collateral to CA1 synapses. We found that age substantially reduces NMDAR-dependent LTP across the spectrum of cognitive outcomes, whereas increased NMDAR-independent LTP occurs distinctively in high-performing aged rats. Moreover, in young rats, behavioral performance correlates strongly with the magnitude of NMDAR-LTP, whereas NMDAR-independent LTP correlates with behavioral performance only in aged rats. Together with similar previous findings on the mechanisms for LTD in this model, these results support the notion that a shift from NMDAR-dependent to NMDAR-independent mechanisms for neural plasticity during aging is associated with better cognitive outcomes.
\end{abstract}

Key words: learning memory; CA1; NMDA receptor; LTP; aging; senility

\section{Introduction}

Aging can have a pronounced impact on mental abilities, particularly on learning and memory. Although such decline is widespread enough to be often considered a normal aspect of aging, some older individuals retain strong cognitive abilities (Cabeza et al., 2002; Davis et al., 2003; Daffner et al., 2006). Hence, current research is focused not only on age-related impairments but also on the neural basis that supports cognition at older ages.

It is widely held that encoding new memories involves adjustments in the strength of connectivity in neural circuits through mechanisms of synaptic plasticity like long-term potentiation (LTP) and long-term depression (LTD) (Bliss and Collingridge, 1993; Bear, 1999). Research on rodent models has successfully identified alterations in LTP and LTD in hippocampal circuits that could contribute to age-related cognitive decline (Landfield and Lynch, 1977; Deupree et al., 1993; Norris et al., 1996; Shankar et al., 1998; Schulz et al., 2002; Tombaugh et al., 2002; Rosenzweig and Barnes, 2003; Burke and Barnes, 2006). Indeed, a number of studies have found correlations between alterations in plasticity at these synapses and severity of impairment in older rats. The status of neural

Received June 25, 2008; revised June 12, 2008; accepted June 16, 2008.

This work was supported by National Institutes of Health Grant P01-AG09973 and Alzheimer's Association Grant IIRG-05-14961. We thank Dr. H.-K. Lee for valuable discussions and comments.

Correspondence should be addressed to either of the following: Michela Gallagher, Department of Psychological and Brain Sciences, The Johns Hopkins University, 105 Ames Hall, 3400 North Charles Street, Baltimore, MD 21218, E-mail:michela@jhu.edu; or Alfredo Kirkwood, Mind/Brain Institute, The Johns Hopkins University, 338 Krieger Hall, 3400 North Charles Street, Baltimore, MD 21218, E-mail: kirkwood@jhu.edu.

DOI:10.1523/JNEUROSCI.2036-08.2008

Copyright $\odot 2008$ Society for Neuroscience $\quad$ 0270-6474/08/288034-06\$15.00/0 plasticity in aged animals with preserved cognitive abilities is less clear. Using a behaviorally characterized rat model, we recently reported that at CA3-to-CA1 synapses in the hippocampus, the NMDA-dependent form of LTD is substantially reduced in all aged rats relative to young adults, but NMDA receptor (NMDAR)-independent LTD is significantly augmented in aged individuals with preserved cognitive ability (Lee et al., 2005). The current study examines whether those findings generalize to the regulation NMDARdependent and -independent forms of LTP in this model.

Two distinct mechanisms of LTP coexist at CA3-to-CA1 synapses (Grover and Teyler, 1990), both of which can be affected by aging. One form of LTP is induced on activation NMDA receptors (NMDAR-LTP), and its induction is reduced in older rats (Rosenzweig and Barnes, 2003; Burke and Barnes, 2006). The other form of LTP requires the activation of L-type voltage-gated calcium channels (VGCC-LTP) and its magnitude is reported to increase with aging (Shankar et al., 1998). We examined the induction of these two forms of LTP in aged individuals representing a full spectrum of cognitive outcomes. We found that the NMDAR-dependent form of LTP is similarly reduced in aged rats with impaired performance and in aged rats with preserved learning ability. In contrast, VGCC-LTP is selectively increased in highperforming aged rats relative to both impaired aged rats and young adults. These findings support the notion that maintenance of cognition in aging may not depend on preserving NMDAR-dependent plasticity, but by compensatory recruitment of NMDAR-independent mechanisms. 


\section{Materials and Methods}

Behavioral assessment. Male Long-Evans rats obtained pathogen-free from Charles River Laboratories were 6 months (young) or 24 months (aged) of age at the time of behavioral characterization for spatial learning in a $1.83-\mathrm{m}$-diameter water maze (opaque water at $27^{\circ} \mathrm{C}$ ). During an $8 \mathrm{~d}$ period, in sessions consisting of three trials a day with a $60 \mathrm{~s}$ intertrial interval, rats were trained to locate a camouflaged platform that remained in the same location $2 \mathrm{~cm}$ below the water surface. During a training trial, the rat was placed in the water at the perimeter of the pool and allowed $90 \mathrm{~s}$ to locate the escape platform. If the rat failed to escape on a trial, it was placed onto the platform and allowed to remain there for $30 \mathrm{~s}$. The position of entry for the animal was varied at each trial. Every sixth trial consisted of a free swim ("probe trial"), which served to assess the development of a spatially localized search for the escape platform. During probe trials, the rat was allowed to swim a total of $30 \mathrm{~s}$ with the escape platform retracted to the bottom of the pool. After $30 \mathrm{~s}$, the platform was raised so that the rat could complete escape on the trial. A "behavioral index," which was generated from the proximity of the rat to the escape platform during probe trials (Gallagher and Nicolle, 1993), was used in correlations with the neurobiological data. This index is the sum of weighted proximity scores measured during probe trials; low scores reflect search near the escape platform, whereas high scores reflect search farther away from the target. "Search error" during training trials refers to the deviation from a direct path to the platform and provided an additional measure for behavioral analysis (Gallagher and Nicolle, 1993).

Electrophysiology. Hippocampal slices were prepared from behaviorally tested young and aged Long-Evans rats. Under deep halothane anesthesia, brains were removed quickly and transferred to the ice-cold dissection buffer containing the following: $212.7 \mathrm{~mm}$ sucrose, $2.6 \mathrm{~mm}$ $\mathrm{KCl}, 1.23 \mathrm{~mm} \mathrm{NaH}_{2} \mathrm{PO}_{4}, 26 \mathrm{~mm} \mathrm{NaHCO}_{3}, 10 \mathrm{~mm}$ dextrose, $3 \mathrm{~mm} \mathrm{MgCl}_{2}$, and $1 \mathrm{mM} \mathrm{CaCl}_{2}$ bubbled with a mixture of $5 \% \mathrm{CO}_{2}$ and $95 \% \mathrm{O}_{2}$. A block of hippocampus was removed and sectioned into $400-\mu \mathrm{m}$-thick slices using a vibratome. The slices were recovered for $1 \mathrm{~h}$ at room temperature in artificial CSF (ACSF): $124 \mathrm{~mm} \mathrm{NaCl}, 5 \mathrm{~mm} \mathrm{KCl}, 1.25 \mathrm{~mm} \mathrm{NaH}_{2} \mathrm{PO}_{4}, 26$ $\mathrm{mm} \mathrm{NaHCO}_{3}, 10 \mathrm{~mm}$ dextrose, $1.5 \mathrm{~mm} \mathrm{MgCl}_{2}$, and $2.5 \mathrm{~mm} \mathrm{CaCl}_{2}$ bubbled with a mixture of $5 \% \mathrm{CO}_{2}$ and $95 \% \mathrm{O}_{2}$. All recordings were done in a submersion recording chamber perfused with ACSF $\left(30 \pm 0.5^{\circ} \mathrm{C} ; 2\right.$ $\mathrm{ml} / \mathrm{min}$ ). Synaptic responses were evoked by stimulating the Schaffer collaterals with $0.2 \mathrm{~ms}$ pulses delivered through concentric bipolar stimulating electrodes (FHC), and recorded extracellularly in CA1 stratum radiatum. Baseline responses were recorded using half-maximum stimulation intensity at $0.033 \mathrm{~Hz}$. NMDAR-LTP was induced by theta burst stimulation (TBS), consisting of four theta epochs delivered at $0.1 \mathrm{~Hz}$. Each epoch, in turn, consisted of 10 trains of four pulses (at $100 \mathrm{~Hz}$ ) delivered at $5 \mathrm{~Hz}$. VGCC-LTP was induced with four $200 \mathrm{~Hz}$ epochs $(0.5$ s) delivered at $0.2 \mathrm{~Hz}$ [in the presence of an NMDA receptor antagonist $(100 \mu \mathrm{M}$ D,L-APV) in the ACSF]. To correlate LTP measurements with the learning index, we averaged the magnitude of LTP from all the slices obtained from one animal to generate the percentage LTP value. The average percentage LTP value was plotted against the respective behavioral index to generate the correlation graphs. To determine the significance of correlation, we performed a linear regression analysis.

\section{Results}

We examined the induction of NMDAR-dependent and NMDAR-independent forms of LTP in the CA3-to-CA1 synapse in hippocampal slices prepared from behaviorally characterized young (6 months of age) and aged (24 months of age) outbred Long-Evans rats. Figure 1 summarizes the results for the behavioral assessment of rats used in this study. During training, there was no age difference in performance on the first trial, before rats had experience with escape to the camouflaged platform in the water maze (Fig. 1, left panel, T1 datapoint). Over the course of training, young rats were more proficient in learning to locate the platform. Analysis of the data in Figure 1, left panel, by two-way ANOVA (age by trial block) revealed improved performance over the course of training (trial block; $F_{(3,71)}=150.13 ; p<$
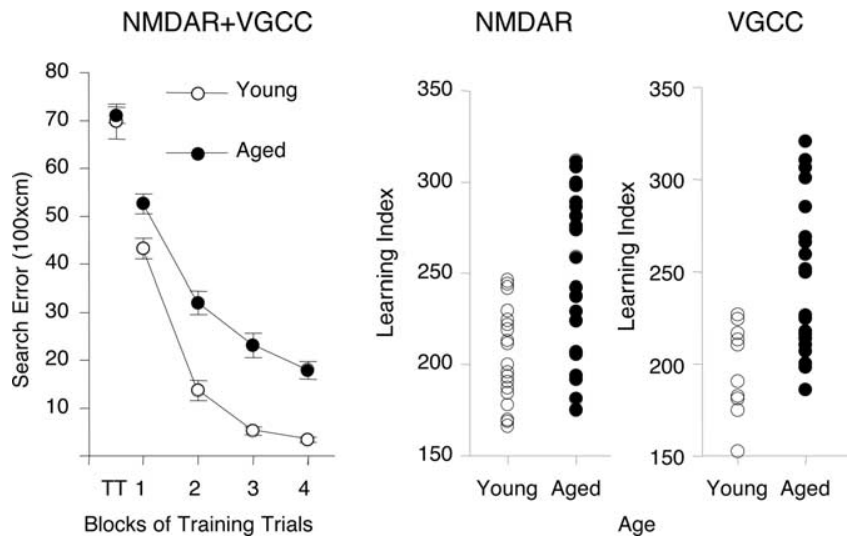

Figure 1. Behavioral characterization of young and aged rats used in the in vitro LTP studies. The left panel shows training trial performance in the Morris water maze for all rats used in this research. Note that age groups do not differ on the first training trial (TT), but young rats are more proficient in learning to escape. Learning index scores were computer from probe trials for each individual rat [for detail, see text and Gallagher et al. (1993)]. Error bars indicate SEM. The middle panel shows the range of performance for young and aged rats used to examine NMDAR-dependent LTP. The right panel shows the range of performance for young and aged rats used to study VGCC-dependent LTP. In both cases, rats that fell within the normative range of young performance (index, $<240$ ) were designated unimpaired, and aged rats with higher scores were designated impaired.

0.0001 ) with a significant difference between the age groups (age; $\left.F_{(1,71)}=51.96 ; p<0.0001\right)$. The trial block by age interaction was not significant $\left(F_{(3,71)}=1.55 ; p=2087\right)$. The learning index scores (Fig. 1, middle and right panels), computed from a key measure of search accuracy during the interpolated probe trials, also differed according to age $(p<0.0001$ and $<0.0021$ for rats used to assess NMDA-dependent and VGCC-dependent plasticity, respectively). In agreement with much previous research in this study population, the aged rats displayed a wide spectrum of outcomes, with many aged rats performing on a par with young adults and substantial numbers outside the entire range of young performance (Fig. 1, middle and right panels). Aged rats that performed outside the range of the young group were designated aged impaired, whereas those that performed on a par with young adults were designated aged unimpaired.

\section{NMDAR-dependent LTP in behaviorally characterized aged rats}

Previous characterizations of LTP in aged rodents indicate that age does not diminish the LTP that can be induced with strong tetanic stimulation, but rather it reduces the LTP magnitude obtained with submaximal stimulation (for review, see Rosenzweig and Barnes, 2003; Burke and Barnes, 2006). Therefore, in these studies, we used theta burst stimulation, an induction protocol that reliably induces LTP (Larson et al., 1986) but does not saturate it (Dudek and Bear, 1992). We also confirmed that this TBS protocol induces only the NMDAR form of LTP in slices prepared from aged rats. As shown in Figure $2 A$, the LTP was fully blocked by the NMDAR antagonist APV (control LTP at 55-60 min after TBS: $133.0 \pm 4.2 \%$ of baseline, $n=5$ slices; LTP in 100 $\mu \mathrm{M}$ APV: $100.0 \pm 2.9 \%, n=4$ slices; $p=0.001$ ). In addition, the LTP induced with TBS was not affected by the L-type channel blocker nifedepine (LTP in $10 \mathrm{~mm}$ nifedepine: $137.9 \pm 12.0 \%$, $n=3$, 10; DMSO control: $135.7 \pm 9.0 \%, n=3,8 ; p=0.888$ ), indicating that VGCC-LTP is not recruited by this protocol in aged rats.

To compare the effects of TBS in the three age groups of be- 


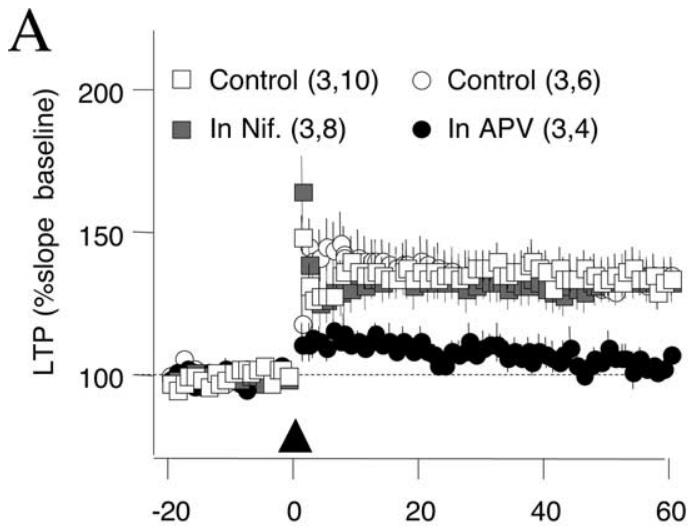

Time after TBS (min)

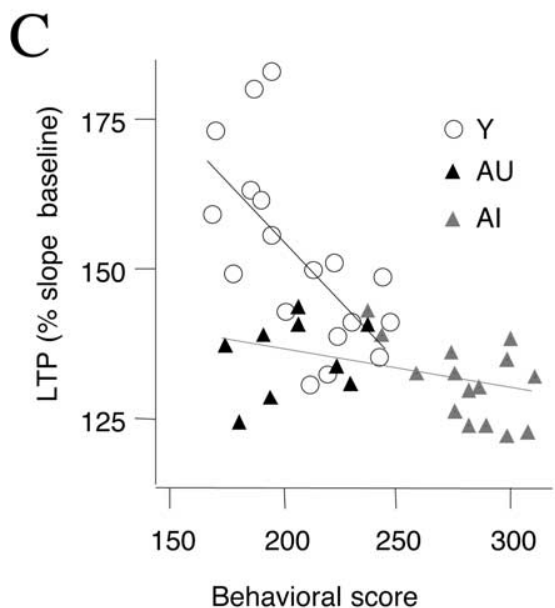

$\mathrm{D}$
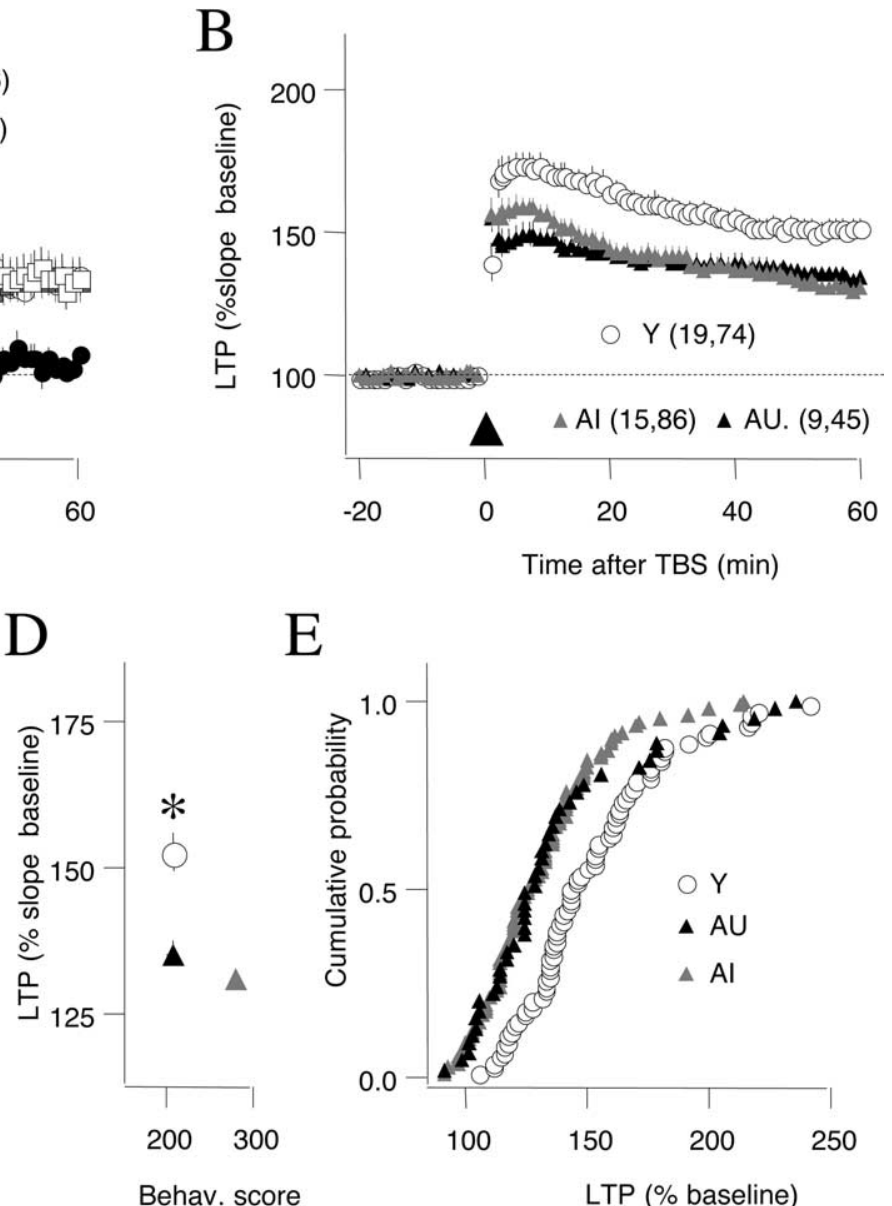

$\mathrm{E}$

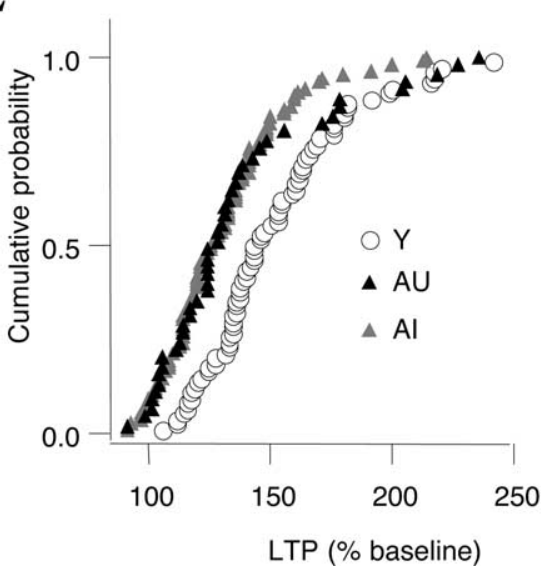

Figure 2. NMDAR-dependent LTP is equally reduced in aged impaired and unimpaired rats. $A$, TBS-induced LTP is blocked by the NMDAR antagonist APV (100 $\mu \mathrm{M}$ ) (control, open circles; APV, filled circles) and not affected by the L-type Ca channel blocker nifedepine (10 $\mu \mathrm{M})$ (DMSO control, open squares; nifedepine, gray squares). $\boldsymbol{B}$, The magnitude of LTP induced by TBS is reduced to a similar extent in aged impaired (gray triangles) and aged unimpaired rats (black triangles) compared with young rats (open circles). Error bars indicate SEM. C, Relationship between behavioral score and LTP magnitude for young, aged impaired, and aged unimpaired individuals. $\boldsymbol{D}$, Summary plot of behavioral scores and LTP averaged across the three age groups. ${ }^{*} p<0.05$. $E$, Distribution of LTP magnitude computed by pooling all the slices in young, Al, and AU rats. The LTP distribution was different between young and either Al (Kolmogorov-Smirnov, $p=0.001)$ or AU ( $p=0.0005$ ), and it was similar between the Al and AU groups $(p=0.61)$.

haviorally characterized rats [young (Y), 17 rats; aged impaired (AI), 15 rats; and aged unimpaired (AU), 9 rats], we first computed the average LTP from all the slices of a given individual (at least three slices per rat; average, $4.8 \pm 0.2$ ). Analysis of those data, shown in Figure $2 B$, confirmed the previously reported reduction of LTP in aged rats (LTP in Y, $152.3 \pm 3.7 \%$; AU, $135.4 \pm 2.1 \%$; AI, $131.0 \pm 1.7 \%$; ANOVA, $F_{(2,40)}=10.6, p=$ $0.0002)$. Interestingly, the results also revealed that the reduction in NMDAR-LTP did not differ in a comparison of aged impaired and aged unimpaired rats ( $p>0.41$, Fisher's test), but each of those subgroups differed significantly from young adults (AI, $p<$ 0.001 ; $\mathrm{AU}, p=0.006$; Fisher's test). These results indicate that a significant reduction in NMDAR-LTP occurs across the spectrum of cognitive outcomes in aging and the occurrence of NMDAR-LTP is not sufficient to distinguish AI and AU rats.

We also examined the relationship between LTP magnitude and behavioral performance (behavioral index score) among the rats in each age group. As shown in Figure $2 C$, in the young group there was a significant correlation between the magnitude of NMDAR-LTP and individual behavioral scores $\left(r^{2}=0.43 ; p=\right.$ 0.003 ), such that better performance was associated with greater LTP. Although a similar trend was evident among rats in the aged group, the correlation was less pronounced $\left(r^{2}=0.162 ; p=\right.$
0.052). Moreover, the slope of the relationship between LTP and behavioral scores was significantly greater for young rats than for older rats $(p<0.001)$. Overall, these data suggest that this type of LTP may not fully account for the behavioral differences among rats in the aged cohort.

\section{VGCC-dependent LTP in behaviorally characterized rats}

The reduced levels of NMDAR-LTP in aged unimpaired rats prompted us to examine the status of VGCC-LTP in aged rats. In juveniles, VGCC-LTP is typically induced with high-frequency stimulation $(200 \mathrm{~Hz})$ in the presence of NMDAR antagonist (100 $\mu \mathrm{M}$ APV) (Grover and Teyler, 1990). We confirmed in slices from aged rats that a protocol of $200 \mathrm{~Hz}$ tetani induced a compound LTP that was only partially reduced by $100 \mu \mathrm{M}$ APV but fully blocked in the presence of APV and the L-type channel blocker nifedepine (Fig. 3A) (control, 148.6 \pm 8.1\%; APV, $128.4 \pm 7.8 \%$; nifedepine plus APV, $\left.104 \pm 1.9 \% ; F_{(2,25)}=3.4 ; p<0.0001\right)$. Using this induction protocol ( $200 \mathrm{~Hz}$ in $100 \mu \mathrm{M}$ APV) in behaviorally characterized rats, we found, as reported previously, that VGCC-LTP is enhanced in aged rats (Shankar et al., 1998). However, this age-dependent increase was restricted to AU rats $(\mathrm{Y}$, $128.3 \pm 3.4 \%, n=10,49 ; \mathrm{AI}, 128.4 \pm 2.1 \%, n=10,54 ; \mathrm{AU}$, $139.6 \pm 2.6 \%, n=10,55 ; F_{(2,27)}=4.9 ; p=0.015$ ) (Fig. 3B). Post 
A

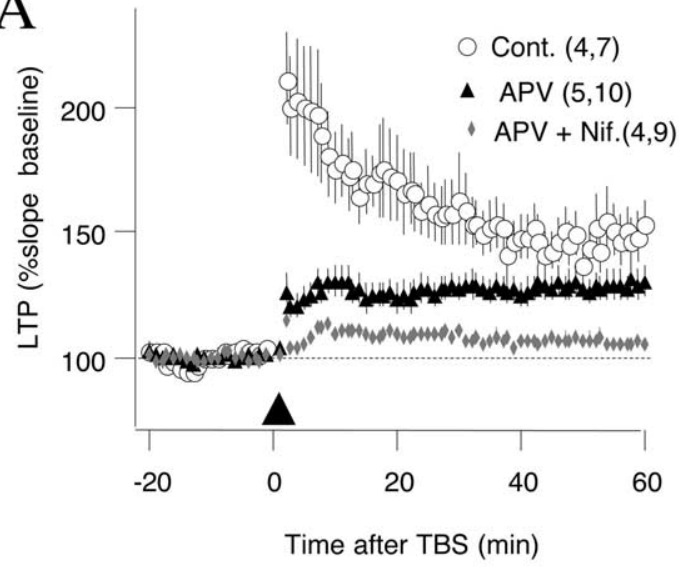

B

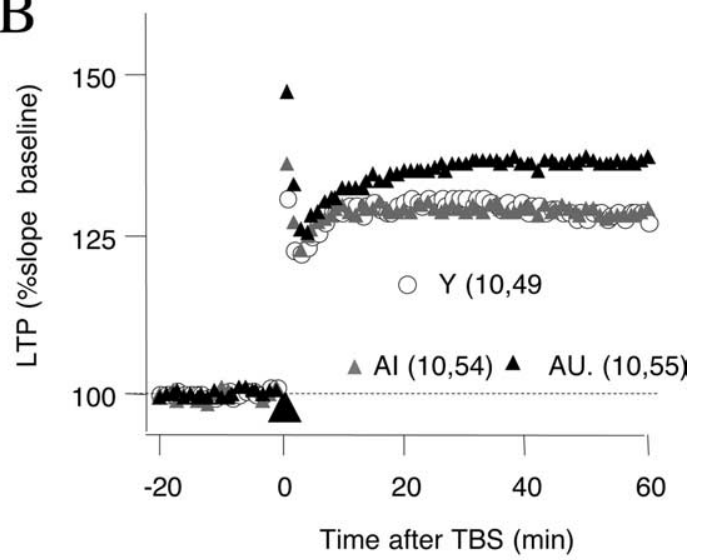

$\mathrm{C}$

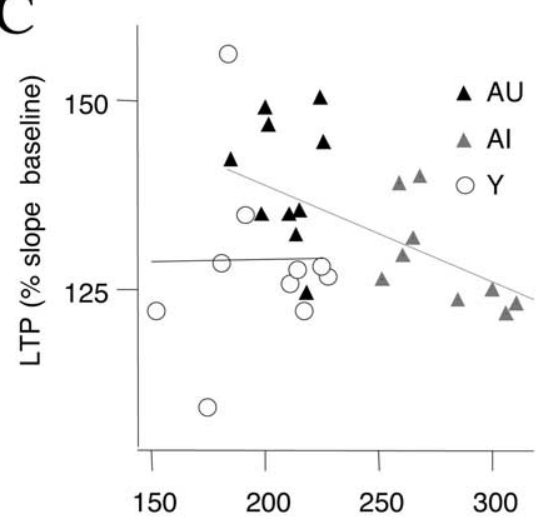

Behavioral score

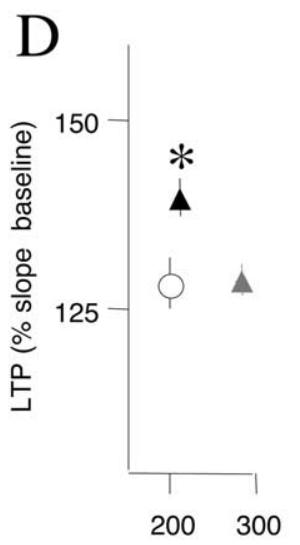

Behav. score
$\mathrm{E}$

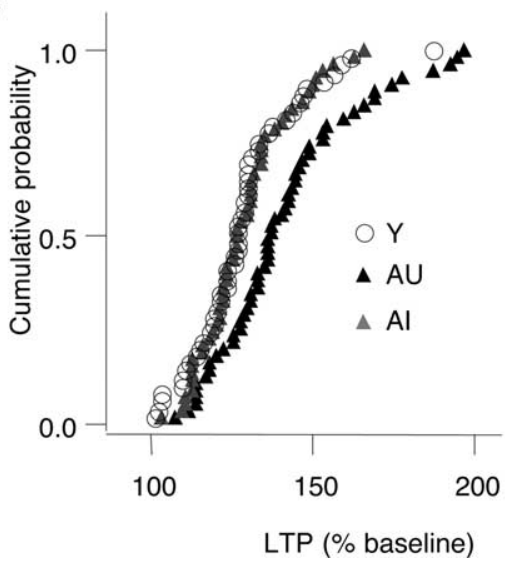

Figure 3. VGCC-dependent LTP is enhanced in aged unimpaired rats. $A, A 100 \mathrm{~Hz}$ tetanus induces a compound LTP that is partially blocked by APV and fully blocked by APV and nifedepine. $B$, The magnitude of LTP induced in the presence of APV is larger in unimpaired rats (black triangles) compared with young (open circles) and aged impaired rats (gray triangles). Error bars indicate SEM. C, Relationship between behavioral score and LTP magnitude for young, aged impaired, and aged unimpaired individuals. D, Summary plot of behavioral scores and LTP averaged across the three age groups. ${ }^{*} p<0.05$. E, Distribution of LTP magnitude computed by pooling all the slices in young, Al, and AU rats. The LTP distribution was different between AU and either Al (KolmogorovSmirnov, $p=0.009)$ or young $(p<0.0085)$, and it was similar between the Al and young groups $(p>0.999)$.

hoc analysis confirmed that VGCC-LTP was not different between the young and the aged impaired groups ( $p=0.96$, Fisher's test), yet each of these groups was different form the aged unimpaired (AI, $p=0.019 ; \mathrm{Y}, p=0.011$ ). An additional analysis of LTP and individual behavioral scores revealed a correlation between LTP magnitude and behavioral index in aged $\left(r^{2}=\right.$ $0.475 ; p=0.0008)$, but not young rats $\left(r^{2}<0.001 ; p=0.978\right)$ (Fig. 3C). As summarized in Figure 3, our results support the notion that an age-dependent increase in VGCC-LTP in some individuals is associated with maintained learning capacity despite the loss of NMDAR-LTP.

\section{Discussion}

The principal findings in our analysis of LTP in aging can be summarized as follows: (1) the induction of NMDAR-LTP was reduced in both aged impaired and unimpaired rats, failing to distinguish those cognitive outcomes; (2) a correlation between NMDAR-LTP magnitude and individual behavioral scores in aged rats was weak, whereas that correlation was robust for young rats; (3) VGCC-LTP was selectively enhanced in aged unimpaired rats; and (4) the magnitude of VGCC-LTP correlated significantly with behavioral scores in aged, but not in young rats. Mechanisms that contribute to altered plasticity at CA3-CA1 synapses have been extensively studied in aged rodents (for review, see Burke and Barnes, 2006), but relatively few previous studies have considered cognitive status (Deupree et al., 1991; Schulz et al., 2002, 2004; Tombaugh et al., 2002). Our results support the notion that the maintenance of cognitive performance at older ages does not necessarily require preservation of NMDAR-LTP but instead may be supported by recruitment of VGCC-LTP. Previously, we described similar changes affecting NMDAR-dependent and -independent forms of LTD at CA3CA1 synapses. Based on these observations, a gain in NMDARindependent forms of plasticity could be a necessary condition to maintain cognitive performance in the face of reduced NMDARdependent forms of plasticity.

Moore et al. (1993) first reported an age-related decrease in LTP at CA1-CA3 synapses when a submaximal induction protocol was used, a finding confirmed by others in subsequent investigations (Worley et al., 1993; Rosenzweig et al., 1997; Shankar et al., 1998). As mentioned, a number of studies have examined differences in LTP at CA3-CA1 synapses as a function of behavioral performance in aged rats. Because LTP protocols in these cases did not isolate NMDA or VGCC-dependent mechanisms, correlations that have indicated greater LTP in unimpaired aged rats relative to those with cognitive impairment could reflect a composite of different forms of plasticity (Deupree et al., 1991; Schulz et al., 2002; Tombaugh et al., 2002). Indeed, Tombaugh et al. (2002) found that the LTP induced in the protocol used in 
their study was not entirely blocked by NMDA antagonist. Our study indicates that substantially less NMDAR-LTP induction occurs across the spectrum of cognitive outcomes in aged LongEvans rats, a model in which many differences in the hippocampal system distinguish impaired rats from aged cohorts with preserved cognitive ability (Colombo et al., 1997; Nicolle et al., 1999; Smith et al., 2000; Wilson et al., 2006). Although a modest correlation of NMDA-LTP with behavioral performance was still evident among the aged rats in our study, a far stronger correlation was obtained in the young adults. Individual differences in NMDAR-LTP, therefore, may not fully account for the behavioral differences among the rats in the aged cohort.

Although reduced NMDA-dependent plasticity is seen across the spectrum of cognitive outcomes, the mechanisms underlying that change could differ among animals in the aged population. Direct measurements of the NMDAR-mediated component of the synaptic responses in CA1 indicate only minimal effects of age (Tombaugh et al., 2002) and only in impaired rats (Lee et al., 2005). Thus, in unimpaired individuals, NMDAR-LTP (Fig. 2 B) and NMDAR-LTD (Lee et al., 2005) are reduced despite a normal NMDAR-mediated synaptic response. We have proposed that synaptic plasticity can uncouple from the activation of NMDARs over the course of aging and that this uncoupling might even serve a neuroprotective function in unimpaired animals against adverse effects associated with excessive NMDAR activation (Lee et al., 2005). Other alterations, seen most prominently in aged rats with behavioral impairment, could play a greater role in the diminished LTP observed in those subjects. For example, an increase in the magnitude of the afterhyperpolarization has been widely reported in aging (Landfield and Pitler, 1984; Norris et al., 1998) and more evident at CA3-CA1 synapses in aged rats with impaired cognitive ability than in unimpaired cohorts (Tombaugh et al., 2005). Thus, reduced excitability during the afterhyperpolarization could contribute to diminished plasticity in the impaired aged subjects.

A previous finding of increased VGCC-LTP in aged rats was based on comparison at different chronological ages without characterization of cognitive status. The important finding here is that significantly greater VGCC-LTP was only found in aged rats that performed on a par with young adults. This may seem somewhat surprising given that age-related calcium dysregulation, including increases in L-type channels and altered homeostasis of intracellular calcium stores, are generally viewed as features contributing to deficits in neurocognitive aging (Campbell et al., 1996; Thibault and Landfield, 1996; Foster and Kumar, 2002). Given evidence for an increase in L-type calcium channel activity in aged rats that perform poorly in some behavioral tasks, it is also not obvious why increased VGCC-LTP was limited to unimpaired rats and not evident in impaired aged animals. One possible account for these findings is that VGCC-LTP depends not only on the activation of L-type channels (Grover and Teyler, 1990) but also on metabotropic glutamate receptors (Little et al., 1995). Thus, enhanced VGCC-LTP induction might only occur in the unimpaired subjects because these aged rats have both increased CA channel functionality and intact mGluR (metabotropic glutamate receptor) signaling, whereas signaling via relevant metabotropic glutamate receptors in the hippocampus is blunted in aged impaired rats (Nicolle et al., 1999). The exact mechanisms that restrict an age-dependent increase of VGCCLTP to unimpaired individuals, however, remain to be determined.

Our analysis, which discriminated between two forms of LTP, revealed that NMDAR-LTP and VGCC-LTP are affected in op- posite ways: the correlation of NMDAR-LTP with behavior is greatly diminished in aged rats, whereas that of VGCC-LTP is increased. These changes might reflect a compensatory adjustment to reduced NMDAR-dependent plasticity, as other mechanisms are recruited to support learning and memory. Such a scenario could have interesting therapeutic implications because cognition decline might not result only from the loss of NMDARdependent plasticity but rather from the failure to compensate for it.

\section{References}

Bear MF (1999) Homosynaptic long-term depression: a mechanism for memory? Proc Natl Acad Sci U S A 96:9457-9458.

Bliss TV, Collingridge GL (1993) A synaptic model of memory: long-term potentiation in the hippocampus. Nature 361:31-39.

Burke SN, Barnes CA (2006) Neural plasticity in the ageing brain. Nat Rev Neurosci 7:30-40.

Cabeza R, Anderson ND, Locantore JK, McIntosh AR (2002) Aging gracefully: compensatory brain activity in high-performing older adults. Neuroimage 17:1394-1402.

Campbell LW, Hao SY, Thibault O, Blalock EM, Landfield PW (1996) Aging changes in voltage-gated calcium currents in hippocampal CA1 neurons. J Neurosci 16:6286-6295.

Colombo PJ, Wetsel WC, Gallagher M (1997) Spatial memory is related to hippocampal subcellular concentrations of calcium-dependent protein kinase $\mathrm{C}$ isoforms in young and aged rats. Proc Natl Acad Sci U S A 94:14195-14199.

Daffner KR, Ryan KK, Williams DM, Budson AE, Rentz DM, Wolk DA, Holcomb PJ (2006) Increased responsiveness to novelty is associated with successful cognitive aging. J Cogn Neurosci 18:1759-1773.

Davis HP, Small SA, Stern Y, Mayeux R, Feldstein SN, Keller FR (2003) Acquisition, recall, and forgetting of verbal information in long-term memory by young, middle-aged, and elderly individuals. Cortex 39:1063-1091.

Deupree DL, Turner DA, Watters CL (1991) Spatial performance correlates with in vitro potentiation in young and aged Fischer 344 rats. Brain Res 554:1-9.

Deupree DL, Bradley J, Turner DA (1993) Age-related alterations in potentiation in the CA1 region in F344 rats. Neurobiol Aging 14:249-258.

Dudek SM, Bear MF (1992) Homosynaptic long-term depression in area CA1 of hippocampus and effects of $N$-methyl-D-aspartate receptor blockade. Proc Natl Acad Sci U S A 89:4363-4367.

Foster TC, Kumar A (2002) Calcium dysregulation in the aging brain. Neuroscientist 8:297-301.

Gallagher M, Nicolle MM (1993) Animal models of normal aging: relationship between cognitive decline and markers in hippocampal circuitry. Behav Brain Res 57:155-162.

Grover LM, Teyler TJ (1990) Two components of long-term potentiation induced by different patterns of affeent activation. Nature 347:477-479.

Landfield PW, Lynch G (1977) Impaired monosynaptic potentiation in in vitro hippocampal slices from aged, memory-deficient rats. J Gerontol 32:523-533.

Landfield PW, Pitler TA (1984) Prolonged $\mathrm{Ca}^{2+}$-dependent afterhyperpolarizations in hippocampal neurons of aged rats. Science 226:1089-1092.

Larson J, Wong D, Lynch G (1986) Patterned stimulation at the theta frequency is optimal for the induction of hippocampal long-term potentiation. Brain Res 368:347-350.

Lee HK, Min SS, Gallagher M, Kirkwood A (2005) NMDA receptorindependent long-term depression correlates with successful aging in rats. Nat Neurosci 8:1657-1659.

Little Z, Grover LM, Teyler TJ (1995) Metabotropic glutamate receptor antagonist, $(R, S)$-alpha-methyl-4-carboxyphenyglycine, blocks two distinct forms of long-term potentiation in area CA1 of rat hippocampus. Neurosci Lett 201:73-76.

Moore CI, Browning MD, Rose GM (1993) Hippocampal plasticity induced by primed burst, but not long-term potentiation, stimulation is impaired in area CA1 of aged Fischer 344 rats. Hippocampus 3:57-66.

Nicolle MM, Colombo PJ, Gallagher M, McKinney M (1999) Metabotropic glutamate receptor-mediated hippocampal phosphoinositide turnover is blunted in spatial learning-impaired aged rats. J Neurosci 19:9604-9610. Norris CM, Korol DL, Foster TC (1996) Increased susceptibility to induc- 
tion of long-term depression and long-term potentiation reversal during aging. J Neurosci 16:5382-5392.

Norris CM, Halpain S, Foster TC (1998) Reversal of age-related alterations in synaptic plasticity by blockade of L-type $\mathrm{Ca}^{2+}$ channels. J Neurosci 18:3171-3179.

Rosenzweig ES, Barnes CA (2003) Impact of aging on hippocampal function: plasticity, network dynamics, and cognition. Prog Neurobiol 69:143-179.

Rosenzweig ES, Rao G, McNaughton BL, Barnes CA (1997) Role of temporal summation in age-related long-term potentiation-induction deficits. Hippocampus 7:549-558.

Schulz D, Huston JP, Jezek K, Haas HL, Roth-Härer A, Selbach O, Luhmann HJ (2002) Water maze performance, exploratory activity, inhibitory avoidance and hippocampal plasticity in aged superior and inferior learners. Eur J Neurosci 16:2175-2185.

Schulz D, Sergeeva OA, Ianovskii E, Luhmann HJ, Haas HL, Huston JP (2004) Behavioural parameters in aged rats are related to LTP and gene expression of ChAT and NMDA-NR2 subunits in the striatum. Eur J Neurosci 19:1373-1383.

Shankar S, Teyler TJ, Robbins N (1998) Aging differentially alters forms of long-term potentiation in rat hippocampal area CA1. J Neurophysiol 79:334-341.
Smith TD, Adams MM, Gallagher M, Morrison JH, Rapp PR (2000) Circuit-specific alterations in hippocampal synaptophysin immunoreactivity predict spatial learning impairment in aged rats. J Neurosci 20:6587-6593.

Thibault O, Landfield PW (1996) Increase in single L-type calcium channels in hippocampal neurons during aging. Science 272:1017-1020.

Tombaugh GC, Rowe WB, Chow AR, Michael TH, Rose GM (2002) Thetafrequency synaptic potentiation in CAl in vitro distinguishes cognitively impaired from unimpaired aged Fischer 344 rats. J Neurosci 22:9932-9940.

Tombaugh GC, Rowe WB, Rose GM (2005) The slow afterhyperpolarization in hippocampal CA1 neurons covaries with spatial learning ability in aged Fisher 344 rats. J Neurosci 25:2609-2616.

Wilson IA, Gallagher M, Eichenbaum H, Tanila H (2006) Neurocognitive aging: prior memories hinder new hippocampal encoding. Trends Neurosci 29:662-670.

Worley PF, Bhat RV, Baraban JM, Erickson CA, McNaughton BL, Barnes CA (1993) Thresholds for synaptic activation of transcription factors in hippocampus: correlation with long-term enhancement. J Neurosci $13: 4776-4786$. 